\title{
Twitter en campaña. Actores mediáticos en Twitter durante la campaña electoral intermedia en México en el año 2015
}

\author{
Salvador Percastre-Mendizábal \\ Universitat Pompeu Fabra \\ salvador.percastre@upf.edu \\ https://orcid.org/0000-0002-5947-51026
}

\section{Twitter in campaign. Actors and electoral discussions on Twitter during the intermediary election campaign in Mexico in 2015}

\section{RESUMEN ABSTRACT}

Desde el enfoque teórico de la Comunicación Política, en este artículo se estudia la actividad de los perfiles de los actores mediáticos (medios de comunicación, periodistas y comunicadores) que intervinieron en las discusiones en

Twitter durante las últimas elecciones intermedias en México en el año 2015. Se realizó un análisis cuantitativo del caso a partir de un esquema metodológico denominado Top Discussion Indicator (Percastre-Mendizábal; Pont-Sorribes; Codina, 2017), el cual permitió la obtención de una muestra

significativa de los mensajes generados durante el día de mayor nivel de interacciones en el periodo de campaña legal.

Como principal aporte al campo de estudio, este artículo analiza las interrelaciones discursivas entre uno de los principales actores clásicos de la comunicación política (Wolton, 1989) pero en el espacio de las redes sociales digitales; además de la aplicación empírica de un modelo de selección de muestras de discusiones políticas en una red de microblogging como Twitter.

PALABRAS CLAVE

Comunicación política, Twitter, Campañas electorales, Actores mediáticos, Elecciones en México
From the theoretical approach of Political Communication, this article studies the activity of the profiles of media actors (media, journalists and communicators) who participated in the discussions on Twitter during the last intermediate elections in Mexico in 2015. A quantitative analysis of the case was made from a methodological scheme called Top Discussion Indicator (Percastre-Mendizábal, Pont-Sorribes, Codina, 2017), which allowed obtaining a significant sample of the messages generated during the day of biggest level of interactions in the legal campaign period. As the main contributions to the field of study, this article analyzes the discursive interrelationships between one of the main classical actors of Political Communication (Wolton, 1989) in the space of digital social networks; in addition to the empirical application of a sample selection model of political discussions in a microblogging network such as Twitter.

KEYWORDS

Political communication, Twitter, Electoral campaigns, Political actors, Mass-media, Elections in Mexico 


\section{Introducción}

De manera creciente, diversos actores del espacio público han encontrado en la Internet un eficaz medio para la comunicación en el contexto electoral. Hoy en día la mercadotecnia política aprovecha los medios digitales con capacidad interactiva, como las redes sociales virtuales y las aplicaciones para los así llamados teléfonos inteligentes, con el objetivo de promover el voto y tratar de influir en la decisión de los ciudadanos.

Pareciera que su intención es que el ciudadano ya no se limite a ser un mero receptor pasivo de mensajes emitidos por partidos y actores políticos a través de los medios de comunicación tradicionales, sino que juegue un papel más activo entablando un diálogo virtual con los emisores preponderantes de los mensajes. De tal modo, los ciudadanos han encontrado nuevas formas de participación política no convencionales y se ha constituido una relación entre interconectividad y política electoral (Di Bonito, 2015), misma que ha captado la atención de la academia en las últimas dos décadas.

Diversos estudios realizados en el ámbito de la comunicación política en el ciberespacio han tenido por objeto de estudio la utilidad de la red de microblogueo (microblogging) Twitter en las campañas electorales (Larsson; Ihlen, 2015; Conway; Kenski; Wang, 2015; Jungherr; Schoen; Jürgens, 2016; Hosch-Dayican; Amrit; Aarts; Dassen, 2016; Park; Park; Lim; Park, 2016). Algunos se han centrado en su uso como medio para informar del contenido de las agendas electorales, reclutar voluntarios o comunicar a los candidatos y partidos con los electores.

Otros análisis se enfocan en el conocimiento de la capacidad de las plataformas digitales para promover la participación política de la ciudadanía más allá del voto durante procesos electorales. Incluso diversas investigaciones (Huberty, 2016; Burckhardt; Duch; Matsuo, 2016; Tsakalidis; Papadopoulos; Cristea; Kompatsiaris, 2015) han llevado más allá su uso y han planteado la predictibilidad de Twitter en política como un elemento novedoso de seguimiento de información para la predicción de resultados electorales en sistemas democráticos. Si bien hay quien afirma (Murthy, 2015) que ha tenido un uso más bien reactivo que predictivo.

Para categorizar en forma general esta corriente de investigación en torno al potencial de los ambientes sociotécnicos interconectados para coordinar acciones de usuarios en línea (Calabrese; Jenard, 2018), particularmente acciones políticas por ejemplo durante una campaña electoral, se han planteado tres líneas de análisis sobre la manera en que Internet puede ser usado: una correspondiente a la acción de informar, otra sobre la de movilizar, y una más acerca de dirigir las acciones electivas (Lilleker; Vedel, 2013). Cada uno de estos aspectos funciona de acuerdo con los sistemas políticos, la cultura cívica y las condiciones electorales específicas de cada caso, tanto a nivel nacional como local.

Así, el presente artículo surge de una investigación desarrollada con el objetivo de describir el uso particular que se hace de la plataforma de microblogueo Twitter, como una herramienta de comunicación política digital en un contexto electoral. Para ello, se estudió el caso de las últimas elecciones intermedias en México llevadas a cabo en el año 2015.

Se realizó un análisis de la actividad de los actores mediáticos, es decir, medios de comunicación, periodistas y comunicadores que intervinieron en las discusiones en Twitter durante las elecciones mexicanas en el año 2015 ya que dicho grupo de atores configura uno de los principales participantes del espacio de la comunicación política tanto digital como no digital. Con un esquema metodológico llamado Top Discussion Indicator (TDI) (Percastre-Mendizábal; Pont-Sorribes; Codina, 2017) que permite la obtención de una muestra significativa en las discusiones generadas durante la campaña legal, se obtuvo una muestra significativa del día en el que se realizaron el mayor número de impactos sobre el tema.

El universo del caso configuró una base de datos que incluye todas las entradas (inputs) en Twitter, es decir tuits y retuits (tweets y retweets) generados en México con la etiqueta (hashtag) \#Elecciones2015, durante el periodo legal de la campaña electoral: del 05 de abril al 03 de junio de 2015. Mientras que la muestra de análisis se delimitó a partir de la obtención de un factor significativo con el TDI, resultando en el día 23 de abril del 2015, como el día de mayor volumen de impactos en la campaña.

\section{Twitter en la campaña electoral}

Durante la última década, la plataforma de interacción digital Twitter, ha jugado un papel predominante en los procesos de comunicación política electoral. Gobiernos, partidos políticos, políticos profesionales y diversos actores públicos han ejercido la comunicación en el ciberespacio a través de la expresión y la acción política, en distintos niveles, entre usuarios de esta red digital (Vaccari, 2015).

Pero, no solo las élites políticas en Twitter (Larrosa-Fuentes, 2017) han hecho uso de esta plataforma; medios de comunicación, periodistas, comunicadores, grupos de presión, y organizaciones de la sociedad civil también han hecho un prolijo uso de este medio social digital. Twitter se ha convertido en una plataforma relevante para la comunicación política, al tiempo que ha crecido de manera innegable en el ámbito del márquetin, por lo que sigue siendo una de las principales redes sociales digitales a nivel global, y un canal poderoso para el desarrollo de comunicaciones de mercadotecnia.

Twitter ha mostrado ser un recurso de alto potencial en campañas políticas digitales en sistemas electorales competitivos 
al rededor del mundo. A través de esta plataforma, los candidatos a puestos de elección popular transmiten sus mensajes político-electorales con el fin de hacerlos llegar de manera sintética y en tiempo real a sus respectivos electorados con la intención de persuadirlos (Hosch-Dayican et al., 2016). La idea es que los medios tradicionales de información los puedan replicar mediante una estrategia comunicativa que combina los medios digitales con los predigitales y así configurar lo que Chadwik (2006) denominó en su momento como ecosistema mediático híbrido.

Twitter se presenta como una herramienta útil a la acción política en términos de su capacidad para erigir figuras públicas y posicionar temas en la agenda (Conway et al., 2015). Lo primero se debe a que ciertos actores de la cibercomunidad de esta plataforma comunicativa tienen capacidad de generar conversaciones a partir de sus publicaciones, lo que eventualmente puede llevar a convertirlos en líderes de opinión. Lo segundo, a través de las etiquetas, que cumplen una función de aglutinar debates en torno a un tema y al mismo tiempo de agrupar temas en torno a algún suceso o evento del mundo en línea (online), pero también del mundo fuera de línea (off line). Con las etiquetas es posible localizar tuits e hilos de conversaciones y delimitar las discusiones con respecto a un tema o temas determinados. Cuando estas se utilizan en una discusión que ha generado un volumen alto de mensajes, se configuran los llamados trending topics o temas del momento. En campañas políticas, estas etiquetas suelen coincidir con asuntos de controversia o incluso devenir en una nueva versión digital de las antiguas consignas de la comunicación predigital.

Analistas de los medios digitales como Kapko (2016), advierten que Twitter ha jugado un papel preponderante durante las recientes elecciones presidenciales en los Estados Unidos. Kapko señala que nunca había sido tan grande la capacidad de un solo tuit de dar forma a la conversación política e impulsar la cobertura de los medios, como hasta ahora.

Si se revisa la distribución de las conversaciones en Twitter alrededor de los debates llevados a cabo durante la campaña presidencial estadounidense entre Donald Trump y Hillary Clinton en octubre de 2016, se descubre que las proporciones fueron $62 \%$ vs $38 \%$, $64 \%$ vs $36 \%$ y $59 \%$ vs $41 \%$, respectivamente, lo que marcó una tendencia a una mayor concentración de información en torno al actual presidente de los Estados Unidos (Statista, 2017a). Una reciente estadística de Statista (2017a) muestra que, en torno a periodos clave de la campaña, por cada tuit automatizado en favor de Clinton, se generó un promedio de 4,9 tuits en favor de Trump. Estos números conducen a pensar -aunque aún sin una sólida base empírica-, que los mensajes políticos a través de Twitter lograrán ampliar la visibilidad de las campañas y devendrán en una mayor penetración social.

Pese al uso extendido de la tuitósfera en los diversos escena- rios de la comunicación política y la excesiva confianza en su efectividad, aún no existe un acuerdo en el mundo académico sobre el efecto real del uso de Twitter en las actitudes y comportamientos de la sociedad. Esto, sobre todo en la respuesta de la ciudadanía ante el tráfico de tuits en tiempos electorales o incluso en escenarios de campaña negativa en el espacio digital (Ceron; d'Adda, 2015).

De manera similar a las conclusiones a las que la academia ha llegado después de casi cien años de investigación de la comunicación de masas, lo que hasta ahora se ha podido confirmar con certeza en cuanto al uso de los dispositivos comunicativos virtuales, al menos en la literatura disponible, es que, algunos medios de comunicación ejercen cierta influencia solo en determinadas personas (Lang; Lang, 1981). Esta premisa puede extenderse a los efectos sociales y políticos de la comunicación digital.

No obstante, el uso intensivo de los medios y redes sociales digitales en las campañas políticas ha repercutido en la generación de enormes cantidades de seguidores (followers), como lo que se observó de manera particular en el caso del uso estratégico de Twitter, en la campaña electoral Donald Trump para presidente de los Estados Unidos de América.

Un reporte de Statista (2017a) muestra que la cuenta @realDonaldTrump tenía 30,13 millones de seguidores en 2017. Con tal cifra, el actual presidente de los EUA ocupa el segundo lugar en seguidores de Twitter para cuestiones políticas, justo debajo de la cuenta aPontifex, perteneciente al Papa Francisco, cuyo alcance es de 33,72 millones. Además, esta plataforma tiene importantes implicaciones en términos de la vigencia de los seguidores; pese a que Barack Obama ya no ocupa la Casa Blanca, su cuenta, @BarackObama, aparece en el tercer lugar del índice mundial, con 96,39 millones de seguidores (Statista, 2017b).

\section{Metodología}

Con una metodología cuantitativa, descriptiva y analítico-interpretativa, se analiza una serie de datos conformados en núcleos conversacionales, delimitados a partir de la etiqueta (hashtag) \#Elecciones2015.

Los datos obtenidos fueron discriminados para ajustarse al caso de estudio, para ello se eliminaron tuits off topic, es decir que, aun cuando contenían dicha etiqueta, no correspondían temáticamente al objeto de análisis. Asimismo, se descartaron aquellos mensajes cuya geolocalización indicara que no habían sido publicados desde México y aquellos que no fueron redactados en idioma español.

La conversación global sobre el caso consta de 191.118 mensajes o inputs (tuits y retuits) a analizar, lo que configura el universo de estudio, el cual se ha delimitado para extraer una muestra de análisis a partir de estos datos. Para realizar 
este proceso de delimitación se ha usado el método de Percastre-Mendizábal; Pont-Sorribes; Codina (2017), denominado Top Discussion Indicator (TDI) que permite establecer cuál es el momento de mayor auge en la discusión en torno a un fenómeno de discusiones en Twitter.

El marco temporal consiste en establecer el día preciso, de entre los días delimitados de duración del caso, en el que se han producido un mayor número de publicaciones, con el objetivo de localizar el momento de mayor volumen de inputs en la discusión. En este caso el marco temporal del fenómeno de comunicación política es el periodo legal de duración de la campaña, que comprendió entre el 5 de abril y el 4 de junio del mismo año, con una duración de 60 días. Siendo el 23/04/2015, el día en que se publicaron el mayor número de impactos: 7.051 .

Una vez aplicado este primer tamiz vinculado al marco temporal, se aplicó un criterio de relevancia para identificar aquellos tuits de mayor impacto de entre los que componen esta muestra. Para obtener los tuits considerados como de alto grado de impacto y que teóricamente tienen mayor relevancia por su potencial impacto comunicativo, en primer lugar, se excluyeron todos los retuits para considerar únicamente a los tuits originalmente emitidos por el usuario o perfil, lo que arrojó un resultado de 6.811 inputs.

De estos tuits originales, fueron seleccionados 621, es decir aquellos que como mínimo tuviesen 10 o más retuits. Este parámetro está establecido como un criterio con unos valores numéricos aleatorios, que sirven de referencia para determinar aquellos mensajes más compartidos en Twitter, ya que en la lógica interna de la línea de tiempo (timeline) de Twitter, los retuits aportan mayor visibilidad a los mensajes que los «me gusta» (like), por lo que su importancia como herramienta de viralización del contenido es más alta. En estudios de casos similares (Percastre-Mendizábal; Pont-Sorribes; Codina, 2017)

\begin{tabular}{|c|c|c|c|c|c|}
\hline Nombre de usuario & Retuits & Me gusta & Seguidores & $\begin{array}{c}\text { No. de } \\
\text { publicaciones }\end{array}$ & $\begin{array}{l}\text { Promedio de «me } \\
\text { gusta» por publicación* }\end{array}$ \\
\hline El Respetable & 4 & 0 & 3.792 .216 & 2 & 0 \\
\hline CNN México & 56 & 52 & 3.101 .329 & 6 & 9 \\
\hline Pedro Ferriz de Con & 11 & 7 & 2.483 .176 & 2 & 4 \\
\hline Pedro Ferriz de Con & & & 1.956 .283 & & \\
\hline Noticias MVS & 82 & 44 & 1.266 .773 & 13 & 3 \\
\hline Periódico Excélsior & 13 & 11 & 1.151 .884 & 3 & 4 \\
\hline Telediario & 2 & 3 & 608.701 & 2 & 2 \\
\hline Notimex & 6 & 2 & 588.225 & 1 & 2 \\
\hline El Financiero & 4 & 1 & 552.313 & 1 & 1 \\
\hline ADNPolítico & 57 & 11 & 389.251 & 6 & 2 \\
\hline 24 HORAS & 38 & 9 & 255.735 & 6 & 2 \\
\hline Periódico Supremo & 1 & 0 & 222.473 & 1 & 0 \\
\hline Periódico Zócalo & 3 & 1 & 158.065 & 3 & 0 \\
\hline Imagen Radio & 1 & 1 & 152.346 & 1 & 1 \\
\hline Mural.com & 9 & 7 & 128.307 & 1 & 7 \\
\hline Politicaonline & 5 & 1 & 121.718 & 3 & 0 \\
\hline Máspormás & 1 & 0 & 104.490 & 1 & 0 \\
\hline Fernando Canales & 10 & 5 & 81.429 & 5 & 1 \\
\hline Plano Informativo & 3 & 0 & 75.535 & 2 & 0 \\
\hline Quadratín Michoacán & 30 & 19 & 68.041 & 5 & 4 \\
\hline Siete24 Noticias & 2 & 0 & 63.914 & 2 & 0 \\
\hline 1070 Radio Noticias & 6 & 2 & 58.616 & 5 & 0 \\
\hline Newsweek en Español & 2 & 3 & 57.985 & 2 & 2 \\
\hline Forbes México & 1 & 0 & 55.738 & 1 & 0 \\
\hline Periódico e-consulta & 12 & 9 & 54.632 & 5 & 2 \\
\hline
\end{tabular}

Tabla1.Actoresmediáticos conmás de50.000 seguidores, duranteel23/04/2015queinteractuaron conlaetiqueta \#Elecciones2015. * Variable proxy de éxito. En sombreado: perfil que presentó una variación importante de seguidores. Elaboración propia. 
se indica que en la mayoría de las veces los tuits obtienen un mayor número de retuits que de favoritos, por lo que resulta pertinente esta relevancia.

De estos 621 tuits se seleccionaron aquellos que fueron emitidos únicamente por actores mediáticos, entre medios de comunicación, periodistas y comunicadores, el día de mayor volumen de mensajes y en el que se manifestaron el mayor número de usuarios en toda la campaña.

Para el análisis se contabilizaron las siguientes variables cuantitativas:

- Seguidores: se señala al número total de seguidores que tenía el perfil el día de análisis del caso.

- Retuits recibidos: en el caso de que el perfil únicamente publicara un tuit sobre el caso de estudio se indica en este apartado los retuits que recibió ese tuit. En caso de que publicara más de un tuit, se sumó el número de retuits que recibieron en total todos los tuits publicados por un mismo usuario, dando como resultado un número total.

- «Me gusta» recibidos: sigue la misma lógica analítica que la de los retuits recibidos, pero en este caso se aplica a los favoritos o «me gusta» recibidos.

- Número de publicaciones: se señala cuántos tuits propios (es decir, tuits originales) publicó un mismo usuario sobre un caso concreto.

- Promedio de «me gusta» por publicación: se refiera a la variable proxy de éxito con la cual se obtuvo el promedio del número de «me gusta» recibidos por publicación del mismo usuario.

\section{Resultados}

Derivado del análisis de los datos generales de la muestra correspondientes al total de los actores mediáticos en México, que interactuaron con la etiqueta \#Elecciones2015 el día 23 de abril de 2015, que es el día con el mayor número de impactos durante la campaña; es posible observar (Tabla 1) que, el sitio web de noticias local con sede en Guadalajara, Jalisco, El Respetable, el prestigiado portal estadounidense de noticias en México CNN México, así como el perfil del famoso periodista mexicano Pedro Ferriz de Con, son los tres perfiles mediáticos que tuvieron la mayor cantidad de seguidores.

De los 24 perfiles con el mayor número de seguidores, entre los que registraron un mayor número de «me gusta», encontramos nuevamente a CNN México, Noticias MVS, y al perfil de la agencia de noticias en el estado de Michoacán, Quadratín Michoacán.

En cuanto al número total de publicaciones hasta el día objeto del análisis de los perfiles, solo Noticias MVS, registró un

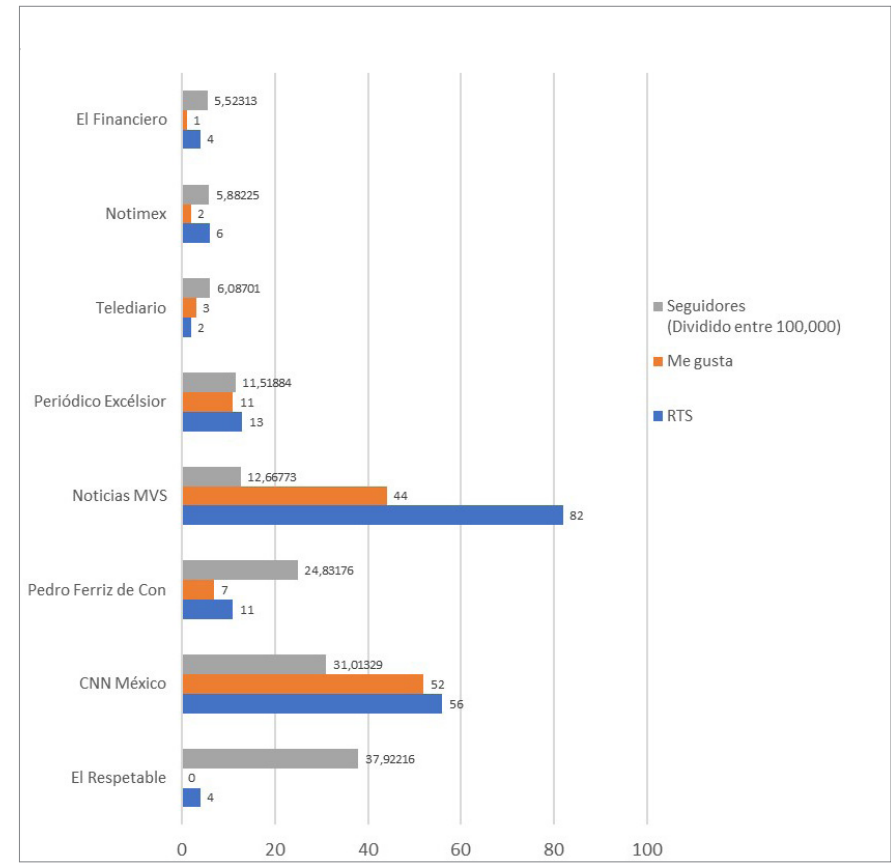

Figura 1. Actores mediáticos relevantes con más de 50.000 seguidores. La cifra de seguidores está dividida entre 100. 000. Elaboración propia.

número considerablemente superior con respecto a los demás perfiles, los cuales registraron menos de 10 publicaciones.

El promedio de «me gusta» entre las publicaciones de estos 24 perfiles, registró una incidencia máxima de 7 en el caso de Mural.com, perfil del sitio web del periódico local del estado de Jalisco, Mural (filial del diario nacional, Reforma). Se debe resaltar que el perfil con el mayor número de seguidores, El Respetable, se encuentra entre los 9 perfiles que registraron 0 en el promedio de 2 «me gusta».

Como se observa en la Figura 1, de entre el total de perfiles mediáticos analizados, en los 5 perfiles con el mayor número

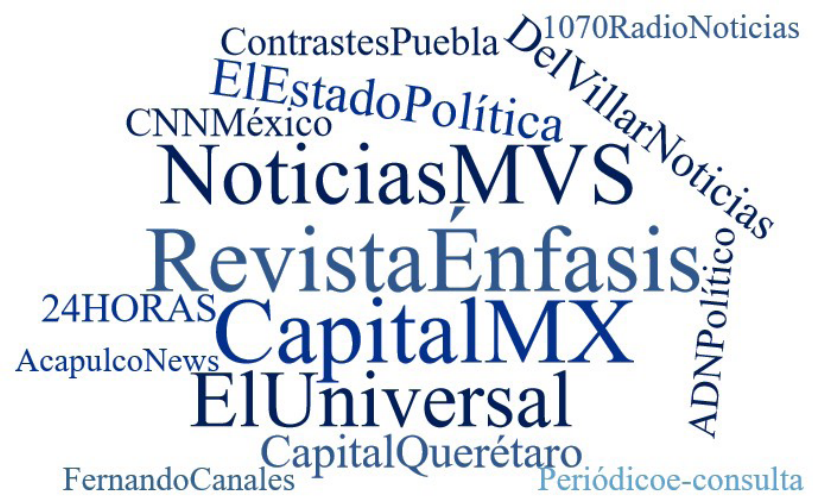

Figura 2. Representación de nube de términos de los actores mediáticos con mayor volumen de tuits, que interactuaron con la etiqueta \#Elecciones 2015. Elaboración propia. 


\begin{tabular}{|l|c|}
\hline \multicolumn{1}{|c|}{ Nombre de usuario } & No. de tuits \\
\hline CapitalMX & 14 \\
\hline Revista Énfasis & 14 \\
\hline Noticias MVS & 13 \\
\hline El Universal & 12 \\
\hline El Estado Política & 8 \\
\hline Capital Querétaro & 7 \\
\hline Del Villar Noticias & 7 \\
\hline 24 HORAS & 6 \\
\hline ADNPolítico & 6 \\
\hline CNN México & 6 \\
\hline Contrastes Puebla & 6 \\
\hline 1070 Radio Noticias & 5 \\
\hline Acapulco News & 5 \\
\hline Fernando Canales & 5 \\
\hline Periódico e-consulta & 5 \\
\hline Quadratín Michoacán & 5 \\
\hline
\end{tabular}

Tabla 2. Actores mediáticos con mayor número de tuits que interactuaron con la etiqueta \#Elecciones 2015. Limitado a actores con 5 o más tuits. Elaboración propia. de seguidores: El Respetable, CNN México, «Pedro Ferriz de Con», Noticias MVS y Periódico Excélsior (perfil del diario nacional generalista Excélsior), existe un elemento común y es que registraron considerablemente un mayor número de retuits que de «me gusta».

Si se considera únicamente la actividad en tuits originales, los perfiles con el mayor número de usuarios el día de análisis fueron el del portal de noticias de la Ciudad de México, CapitalMX, el de la Revista Énfasis (cuya cuenta ha sido suspendida), el de Noticias MVS y el del diario generalista nacional con el mayor número de lectores en México, El Universal; cuyos perfiles registraron más de 10 tuits. Ver Tabla 2.

Como se aprecia en la Figura 3, donde se representan los perfiles mediáticos con más de 10 retuits recibidos de entre el total de 130 perfiles analizados, los medios que más impacto y mayor empatía generaron al ser compartidos en un mayor número de ocasiones fueron el sitio web de noticias local del estado de Oaxaca, Oaxaca Político, El Universal, el sitio web de noticias locales en el estado de Veracruz, Clarín Veracruzano y el perfil sobre noticias y redes sociales, INFOMOVIL; que registraron más de 100 retuits individualmente.

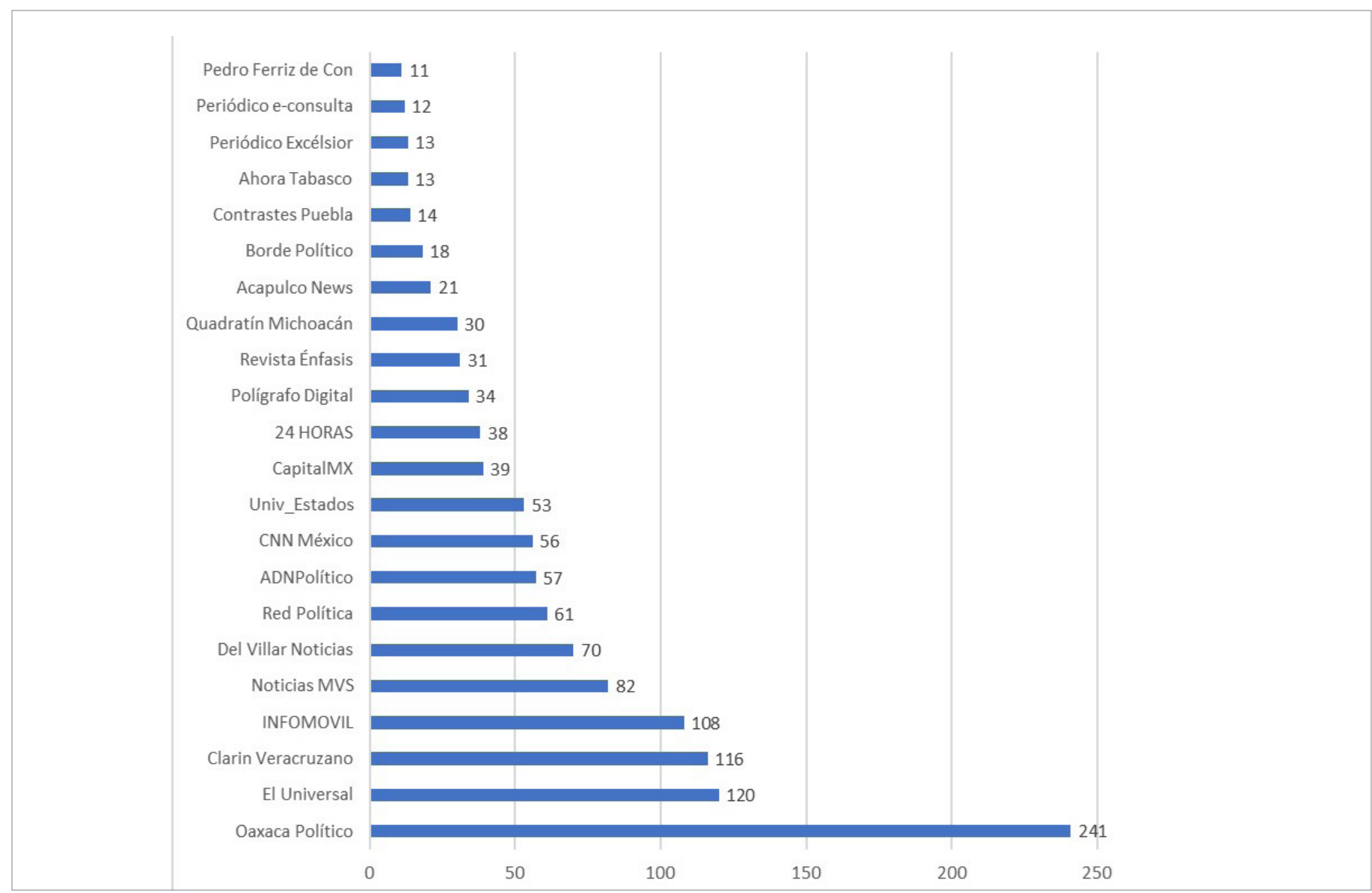

Figura 3. Actores mediáticos cuyos tuits tuvieron mayor cantidad de retuits recibidos. Limitado a actores con más de 10 retuits. Elaboración propia. 


\section{Debate y conclusiones}

Debido a que algunos perfiles presentan una crecimiento significativo en la cantidad de seguidores durante el transcurso del día de análisis, ello permite comprobar que ciertos perfiles con un elevado número de seguidores tuvieron un aumento significativo de followers el día de mayor tráfico de mensajes durante la campaña, lo que confirma que: (1) efectivamente el día de mayores discusiones es el de la muestra obtenida con el método del TDI y que (2) presumiblemente es también ese día, el día más álgido de discusiones en torno a ese fenómeno de comunicación política, el que registra un aumento significativo de seguidores en perfiles mediáticos.

La mayoría de los perfiles mediáticos en el momento de mayor volumen de información contaba con un bajo número de seguidores, un número marginal de perfiles siguiéndolos entre ellos, y apenas contaba con algunos retuits en sus publicaciones, lo que permite inferir que ese día, el de mayor conversación, únicamente los perfiles con un número elevado de seguidores son los potencialmente susceptibles de ganar más seguidores.

Por otro lado, los perfiles con el mayor número de retuits, fueron perfiles de -o vinculados a- medios de comunicación tradicionales como Noticias MVS y CNN México (TV), o ADNPolítico (revista). Lo que presumiblemente sea el resultado de los esfuerzos de los medios tradicionales por no perder su hegemonía en el ecosistema mediático de las redes sociales digitales.

Un indicador relevante para el análisis de conversaciones en Twitter es sin duda los retuits recibidos, ya que existe una mayor probabilidad de que aquel o aquellos usuarios que retuitean (retweeting) algún contenido de algún otro usuario, coincida o esté de acuerdo con el contenido del mensaje reposteado (reposting), lo que permite inferir que a mayor cantidad de retuits recibidos de los mensajes emitidos por un usuario, mayor será el impacto entre los perfiles unidos en la conversación a través de la etiqueta mencionada.

Es posible observar una variación entre las cuentas de actores mediáticos con mayor cantidad de tuits y las cuentas con mayor cantidad de retuits. Esto puede ser indicador de una reacción por parte de los usuarios de la plataforma ante el contenido de los tuits, no correlacionada directamente con el número de seguidores.

Finalmente, el análisis de este caso de estudio situado en el año 2015, apenas 5 años después del surgimiento de la versión en español de Twitter, demuestra que ya en ese momento, esta plataforma había despertado un profundo interés en la opinión pública digital en México, para explorar los usos y aportaciones específicos de esta herramienta en el contexto electoral. Además, como se demuestra aquí, los medios tradicionales (legacy media) y especialmente los medios preponderantes (mass-media) han intentado desde entonces posicionarse en los espacios deliberativos y de discusión de los asuntos públicos, como son -ahora- las redes sociales digitales.

Así, la hipótesis de la presencia de los actores mediáticos en una campaña electoral como uno de los tres grupos de actores clásicos de la comunicación política se ve confirmada, ahora en un ecosistema digital. Sin embargo, aún queda para futuras investigaciones el análisis del comportamiento de los otros dos grupos de actores intervinientes en un fenómeno de comunicación política: políticos y ciudadanos. Queda para futuras investigaciones el estudio específico del papel que juegan estos actores y sus implicaciones para la vida democrática de una sociedad.

\section{Referencias}

Burckhardt, P., Duch, R., y Matsuo, A. (2016). Tweet as a Tool for Election Forecast: UK 2015 General Election as an Example. Recuperado de https://asiapolmeth.princeton.edu/sites/default/files/polmeth/ files/uk_election_tweets_asia_polmeth.pdf

Calabrese, L., y Jenard, J. (2018). Talking about News. A Comparison of readers' comments on Facebook and news websites. French Journal for Media Research, 10. Recuperado de http://frenchjournalformediaresearch.com/lodel/index.php?id=1684

Ceron, A., y d'Adda, G. (2015). E-campaigning on Twitter: The effectiveness of distributive promises and negative campaign in the 2013 Italian election. New Media \& Society, 18(9), 1935-1955. https://doi. org/10.1177/1461444815571915

Chadwick, A. (2006). Internet politics: States, citizens, and new communication technologies. Nueva York: Oxford University.

Conway, B., Kenski, K., y Wang, D. (2015). The rise of Twitter in the political campaign: Searching for intermedia agenda-setting effects in the presidential primary. Journal of computer-mediated communication, 20(4), 363-380.

Di Bonito, I. (2015). Comunicación política, partidos y nuevo entorno digital. Estudio de las campañas electorales catalanas de 2010 y 2012 en la red. Hipertext.net, (13). Recuperado de http://raco.cat/index. php/Hipertext/article/view/298416/389444

Hosch-Dayican, B., Amrit, C., Aarts, K., y Dassen, A. (2016). How Do Online Citizens Persuade Fellow Voters? Using Twitter During the 2012 Dutch Parliamentary Election Campaign. Social Science Computer Review, 34(2), 135-152.

Huberty, M. (2015). Can we vote with our tweet? On the perennial difficulty of election forecasting with social media. International Journal of Forecasting, (31), 992-1007.

Jungherr, A., Schoen, H., y Jürgens, P. (2016). The mediation of politics through Twitter: An analysis of messages posted during the campaign for the German federal election 2013" Journal of computer-mediated communication, 21(1), 50-68.

Kapko, M. (2016). Twitter's impact on 2016 presidential election is unmistakable. Recuperado de https://www.cio.com/author/MattKapko

Lang, G.; Lang, K. (1981). Watergate: An exploration of the agenda-building process. En G. Wilhoit, y H. de Bock (eds.), Mass Communication Review Yearbook 2. Beverly Hills: Sage. 
Larrosa-Fuentes, J. S. (2016). Deliberation and Conversation between Political Elites and Social Media Users during Guadalajara's Elections: a Political Communication Systems Approach. Trípodos, (39), 109-125.

Larsson, A., y Ihlen, $\emptyset$. (2015). Birds of a feather flock together? Party leaders on Twitter during the 2013 Norwegian elections. European journal of communication, 30(6), 666-681.

Lilleker D., y Vedel T. (2013). The Internet in Campaigns and Elections. En W. Dutton (ed.), The Oxford Handbook of Internet Studies. Oxford: Oxford University Press.

Murthy, D. (2015). Twitter and elections: are tweets, predictive, reactive, or a form of buzz?. Information, Communication $\&$ Society, 18(7), 816-831.

Park, S. J., Park, Ji. Y., Lim, Y. S., y Park, H. W. (2016). Expanding the presidential debate by tweeting: The 2012 presidential election debate in South Korea. Telematics and Informatics, (33), 557-569.

Percastre-Mendizábal, S., Pont-Sorribes, C., y Codina, L. (2017). A sample design proposal for the analysis of Twitter in political communication. El profesional de la información, 26(4), 579-588.

Statista (2017a). Numbers of automated pro-Trump tweets for every automated pro-Clinton tweet around select key periods of the U.S. presidential election campaign in 2016. Recuperado de https://www. statista.com/statistics/699668/us-pro-trump-bot-tweets-duringcampaign

Statista (2017b). World leaders with the most Twitter followers as of may 2017. Recuperado de https://www.statista.com/statistics/281375/heads-of-state-with-the-most-twitter-followers

Tsakalidis, A., Papadopoulos, S., Cristea, A. I., y Kompatsiaris, I. (2015). Predicting Elections for Multiple Countries Using Twitter and Polls. IEEE Computer Society, (marzo/abril), 1541-1672.
Vaccari, C., Valeriani, A., Barberá, P., Bonneau, R., Jost, J. T.. Nagler, J., y Tucker, J. A. (2015). Political Expression and Action on Social Media: Exploring the Relationship Between Lowerand Higher-Threshold Political Activities Among Twitter Users in Italy. Journal of Computer-Mediated Communication, (20), 221-239.

Wolton, D. (1989). La Communication politique: Construction d'un model. Hermès, (4), 27-42.

\section{CV}

Salvador Percastre-Mendizábal. Es investigador doctoral en comunicación política, del Grup de Recerca en Comunicació Política, Mitjans i Democràcia de la Universitat Pompeu Fabra, Barcelona y adscrito al ReSIC de la Université Libre de Bruxelles (Bélgica). Docente de posgrado en la UPF Barcelona School of Management y en el ICPS-Universitat Autònoma de Barcelona. Miembro de la European Communication Research and Education Association y de la Asociación Latinoamericana de Investigadores en Campañas Electorales. Ha realizado estancias de posgrado en la Universidad Complutense de Madrid y en la UNAM (México) y ha sido becario Conacyt-Cocit. Es maestro en Estudios del Desarrollo Global en la especialidad en ciencia política y licenciado en Ciencias de la Comunicación en la especialidad en comunicación política. http://www.directorioexit.info/ficha4406

\section{осм Observatorio deCibermedios}

\author{
https://observatoriocibermedios.upf.edu/ \\ upf. $\begin{array}{ll}\text { Universitat } & \text { Departamento } \\ \text { Pompeu Fabra } & \text { de Comunicación }\end{array}$ \\ upf. $\begin{array}{ll}\text { Pompeu Fabra } & \begin{array}{l}\text { de Comunicación } \\ \text { Barcelona }\end{array} \\ \text { Grupo DigiDoc }\end{array}$

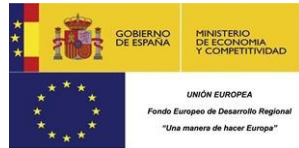

El Observatorio de Cibermedios es una producción del Grupo de Investigación en Documentación Digital y Comunicación Interactiva (DigiDoc) del Departamento de Comunicación de la Universitat Pompeu Fabra.

El Observatorio de Cibermedios (OCM) forma parte del proyecto del Plan Nacional "Creación y contenido interactivo en la comunicación de información audiovisual: audiencias, diseño, sistemas y formatos". CS02015-64955-C4-2-R (MINECO/ FEDER), Ministerio de Economía y Competitividad (España). 